\title{
Surgical Principles in the Treatment of Ulcerative Colitis
}

\author{
Florian Kühn Ernst Klar \\ Department of General, Thoracic, Vascular and Transplantation Surgery, University of Rostock, Rostock, Germany
}

Keywords

Ulcerative colitis · Surgery · IPAA · Pouchitis

\section{Summary}

Background: The primary treatment of ulcerative colitis (UC) is conservative; surgical intervention is carried out in the case of therapy-refractory situation, imminent or malignant transformation, or complications. Surgery for UC should be indicated by interdisciplinary means. Despite the development of drug therapy - in particular the introduction of biologics -, a surgical intervention becomes necessary in a relevant proportion of patients with UC throughout lifetime. Methods: A selective literature search was conducted, taking into account the current studies, reviews, meta-analyses, and guidelines. PubMed served as a database. The present work gives an overview of the surgical options, outcome as well as peri- and postoperative management for patients with UC. Results: Approximately $20 \%$ of patients with UC will require surgery during the course of their disease. The rate of colectomy after a disease duration of 10 years is at approximately $16 \%$. Unlike Crohn's disease, UC is principally surgically curable since it is naturally limited to the colon and rectum. Restorative proctocolectomy with an ileal pouch-anal anastomosis represents the surgical treatment of choice. Large studies show a postoperative complication rate of around $30 \%$ and a low mortality of $0.1 \%$ for this procedure. Chronic pouchitis is one of the main factors limiting the surgical success of curing UC. Despite a high postoperative complication rate, there is a long-term pouch success rate of $>90 \%$ after 10 and 20 years of follow-up. Conclusion: A close cooperation between the various disciplines in the pre- and postoperative setting is essential for an optimal outcome of patients with UC. Despite a $30 \%$ rate of early postoperative complications, normal quality of life can ultimately be reached in more than $90 \%$ of patients in experienced centers.

(c) 2015 S. Karger GmbH, Freiburg

\section{Introduction}

The prevalence of ulcerative colitis (UC) has been reported to be as high as 249 per 100,000 persons in North America and 505 per 100,000 persons in Europe [1]. Approximately 20\% of patients with UC will require surgery during the course of their disease. The rate of colectomy after a disease duration of 10 years is at approximately $16 \%$ [2]. Unlike Crohn's disease, UC is principally curable by surgery since its manifestation is basically restricted to the colon and rectum. The operative treatment of choice is restorative proctocolectomy with ileal pouch-anal anastomosis (IPAA), guaranteeing the complete removal of the substrate of disease down to the dentate line with restoration of the continuity. A therapy-refractory course is considered to be the most common indication for surgery, followed by dysplasia or manifest neoplasia. Perforation, fulminant bleeding, and toxic megacolon represent the emergency indications for surgery in patients with UC.

\section{Emergency Indication (Bleeding, Fulminant Flare/ Toxic Megacolon)}

The incidence of severe bleeding in patients with UC amounts to approximately $4.5 \%$ [3]. Indication for surgery is considered either for a massive initial bleeding with hemodynamic instability and need for catecholamines or if a patient requires more than 4 red cell concentrates per $24 \mathrm{~h} \mathrm{[3]}$. The diagnosis of a refractory fulminating flare is established in an interdisciplinary fashion. The radiological criterion for a toxic megacolon is a colonic dilation $>6$ $\mathrm{cm}$ [4]. The intractable fulminant flare represents an urgent indication for surgery if a substantial improvement cannot be achieved by means of intensive care treatment in conjunction with specific anti-inflammatory drug therapy over a period of $72 \mathrm{~h}$ [3]. A main challenge for the surgeon is to recognize an inadvertent progression of sepsis, the clinical signs of which are often suppressed by high-dose corticoids and antibiotics.

\section{KARGER \\ Fax +497614520714




\section{Refractory Course and Corticosteroid Resistance}

A treatment-refractory course, despite the use of immunosuppressive drugs including biologicals, represents an absolute indication for surgery. Similarly, the patient should be operated on if corticoid dependence is obvious, i.e. the corticoid medication cannot be lowered below the Cushing threshold of $7.5 \mathrm{mg}$ of prednisolone equivalent daily. From this course of the disease, which comprises weeks or months, the intractable fulminant flare must be distinguished which cannot be controlled even by high doses of corticosteroids ('corticosteroid-resistant') and requires emergency surgery.

\section{Risk of Cancer and Dysplasia}

Compared to the general population, the risk of colorectal cancer is increased in patients with UC [5]. Accordingly, a surveillance colonoscopy should be performed annually in patients with pancolitis and a disease duration $\geq 8$ years. Patients with a left-sided or distal colitis should be submitted to surveillance colonoscopy every 1 or 2 year(s) after a disease duration of 15 years [3].

In case of a histologically proven colitis-associated high-grade intraepithelial neoplasia (IEN)/dysplasia a proctocolectomy is indicated after the diagnosis has been confirmed by an external pathologist. For resection of manifest colitis-associated cancer, the same principles apply as for the oncological resection of sporadic colorectal cancer.

In the presence of a low-grade IEN/dysplasia in flat mucosa also confirmed by an external pathologist - either proctocolectomy (relative indication for surgery) or an endoscopic biopsy control within 3 months should be offered to the patient [3]. The patient has to be informed of the risk of malignancy. In this situation, other centers and we advise the patient to undergo surgery because a 'sampling error' represents a significant risk of missing a highgrade dysplasia or carcinoma in repeated colonoscopies.

\section{Surgical Treatment of Ulcerative Colitis}

Restorative proctocolectomy with IPAA is the operation of choice for patients with UC; it represents the only technique by which the substrate of the disease is completely removed and gastrointestinal continuity is reestablished. It is important to include the rectal mucosa down to the dentate line since residual tissue in this location may give rise to intractable pouchitis later on. Patients after IPAA can discontinue colitis-related medications, particularly immunosuppressants and immunomodulators, thereby avoiding associated adverse effects. In addition, the procedure substantially reduces the risk for dysplasia or cancer to the pouch itself in the rare cases where severe chronic pouchitis cannot be controlled.

The operation will be carried out as a one-, two- or three-stage procedure - depending on the individual risk profile of the patient. In general, the two-stage procedure with a protective ileostomy

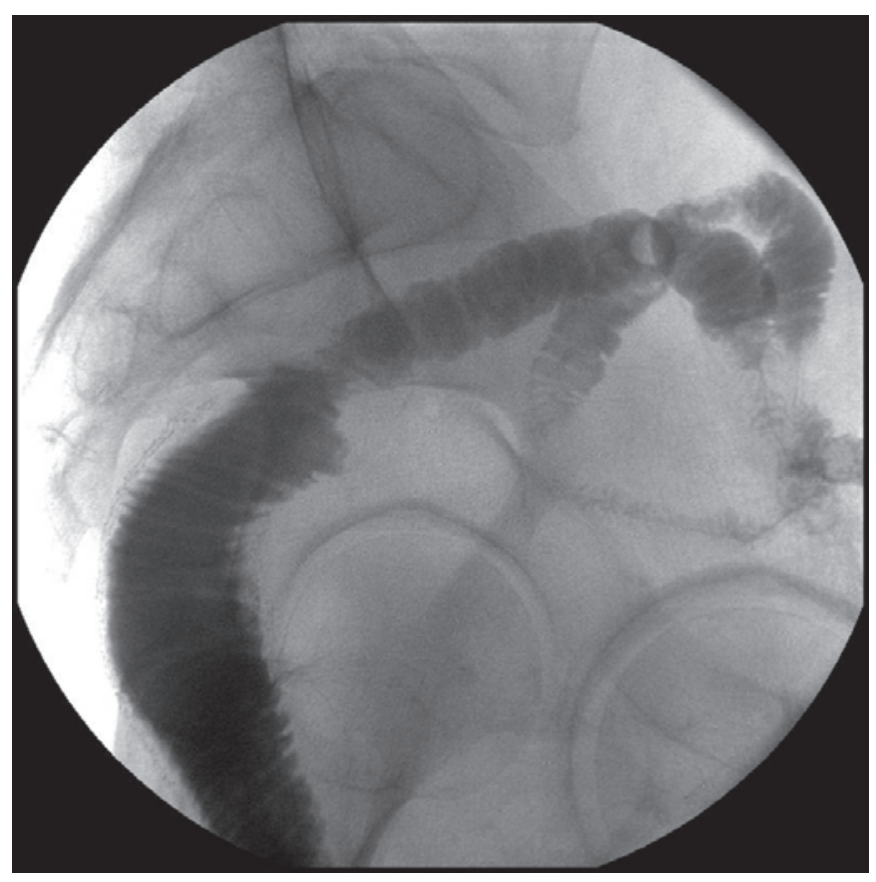

Fig. 1. Regular pouchography (lateral) before closure of ileostomy.

represents the most common variant. A three-stage procedure is conducted for emergency indications, in patients with a considerably reduced general health condition, or in patients depending on high doses of immunosuppressive therapy. The first step includes subtotal colectomy and terminal ileostomy with Hartmann stump formation. Secondly, after consolidation of the patient, the remaining proctectomy with IPAA and protective ileostomy is conducted. The loop ileostomy is then taken down in the third step. Before closure of the ileostomy, a pouchoscopy and pouchography should usually be performed to rule out stenosis, fistulas, or an inflammation ('pouchitis'). Figure 1 shows a regular pouchography before takedown of the ileostomy. Preoperative evaluation and documentation of sphincter function is necessary, e.g. by means of sphincter manometry.

In recent years, there has been a trend towards performing a three-step procedure [6]. The reason for this seems to be an intensified conservative therapy with increasing use of biologics. This entails the risk of surgical complications due to an often significantly reduced general condition of the patient. The three-stage procedure offers a better control in these cases. In contrast, a onestage operation should be reserved only for young patients with good sphincter function, low activity of inflammation, good general condition, low-dose immunosuppressive therapy, and a totally tension-free IPAA. In our series this ideal condition is met in less than $10 \%$ of all patients.

The configuration of the J-pouch should provide a volume of 100-125 ml, with a length of about $15 \mathrm{~cm}$ (fig. 2). Before installation of the pouch-anal anastomosis transanal mucosectomy is necessary as the last step of the monobloc resection in order to achieve a complete removal of the substrate of disease (fig. 3). If a tensionfree anastomosis cannot be obtained due to an elevated root of 
mesentery, mesenteric extension by corresponding windowing of the mesentery has to be performed. Here, special surgical experience is necessary in order to prevent hypoperfusion of the pouch.

According to the German AWMF guideline on UC, restorative proctocolectomy with IAPP can be performed laparoscopically - in experienced centers - as an alternative to open surgery. A recent meta-analysis of 2,428 patients demonstrated a longer operating time with less postoperative wound infections and a shorter hospital stay for the laparoscopic approach. The complication rates and long-term outcomes were equivalent [7].

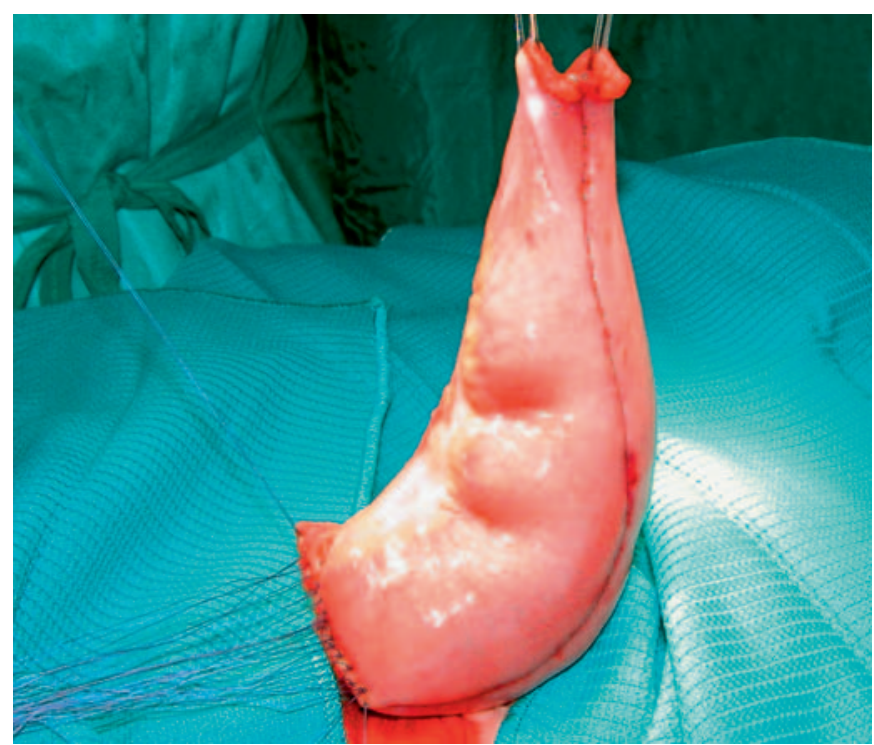

Fig. 2. Ileal J-pouch (volume: 100-125 ml).

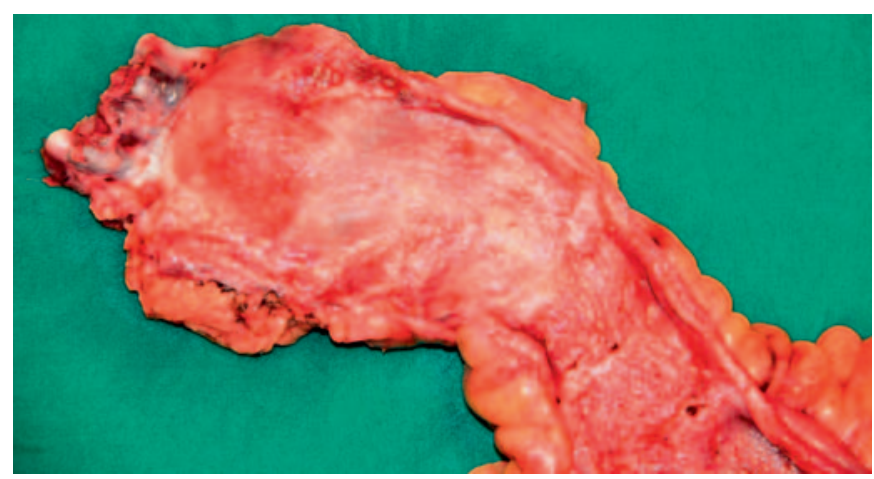

Fig. 3. Proctocolectomy specimen (rectal part) after transanal mucosectomy including the dentate line in order to achieve a complete removal of the substrate of disease.

\section{Morbidity and Mortality}

The IPAA is a challenging surgical procedure with relevant morbidity rates also in experienced hands but low mortality. Large studies have shown postoperative complication rates of approximately $30 \%$ with a mortality rate of $0.1 \%$ [8]. In our own patient population, we revealed a perioperative complication rate of $33.3 \%$ in the absence of mortality (table 1). For ileoanal pouch surgery in UC a center effect was demonstrated with a significant decrease in the rate of complication [9].

\section{Pouchitis and Follow-up}

An idiopathic primary pouchitis occurs in up to $50 \%$ of patients with UC during the first 10 years after surgery and accounts for up to $10 \%$ of pouch failures $[10,11]$. Although the etiology of pouchitis remains unclear, there is significant clinical evidence that the microbial community plays an important role in its pathogenesis. Risk factors, genetic associations, and serological markers of pouchitis suggest that the interactions between the host immune responses and the pouch microbiota underlie the etiology of this idiopathic inflammatory condition [12]. The symptoms of pouchitis can include increased frequency of bowel movements, bleeding, urgency, abdominal cramping, and tenesmus during pouch evacuation. The diagnosis of pouchitis is based on the presence of symptoms as well as endoscopic and histological evidence of inflammation of the pouch. An overlap with UC is suggested by the frequency with which pouchitis affects patients with UC as underlying disease compared to patients with familial adenomatous polyposis (FAP). At present, antibiotics and local corticosteroid enemas (foam) are the most effective treatment for pouchitis [13, 14]. Chronic pouchitis is one of the main factors that limit the surgical success of curing UC. However, a high quality of life has been documented 10 and 20 years after IPAA in more than $90 \%$ of patients [15]. Chronic pouchitis is considered a precancerous lesion with the potential of malignant transformation after long duration of disease ( $>10$ years). To detect and treat pouchitis accordingly, patients have to take part in a lifelong follow-up program. The intervals of pouchoscopy with histological examination are based on the existence and intensity of chronic pouchitis and range from 6 months to 2 years.
Table 1. Mortality und complication rate after IPAA

\begin{tabular}{lllll}
\hline Author & $\begin{array}{l}\text { Mortality, } \\
\%\end{array}$ & $\begin{array}{l}\text { Complication rate } \\
\text { (Clavien }>\text { III), } \%\end{array}$ & $\begin{array}{l}\text { Pouchitis, } \\
\%\end{array}$ & $\begin{array}{l}\text { Pouch failure, } \\
\%\end{array}$ \\
\hline $\begin{array}{l}\text { Fazio et al., 2013 [8] } \\
\text { Michelassi et al., 2003 [23] }\end{array}$ & 0.1 & 33.5 & 33.8 & 4 \\
$\begin{array}{c}\text { McGuire et al., 2007 } \\
\quad \text { Review) [24] }\end{array}$ & 0.25 & 30.1 & - & 3.4 \\
$\begin{array}{c}\text { Department of Surgery, } \\
\text { University of Rostock }\end{array}$ & 0 & 30 & $20-59$ & $3-12$ \\
\hline
\end{tabular}




\section{Quality of Life and Long-Term Results after Ileal Pouch-Anal Anastomosis}

An IPAA achieves the best possible quality of life for the patients - with an average of 5-6 bowel movements per day and the preservation of continence in $90 \%$. Several studies have shown quality of life levels after IPAA which are comparable with the general population [16]. However, the occurrence of perioperative complications will significantly affect the quality of life in these patients. Despite high postoperative complication rates, a long-term success rate with a good quality of life was shown in $>90 \%$ after 10 and 20 years after IPAA [15]. In our center, out of a total of 57 patients, only $3.5 \%$ experienced a pouch failure with need for permanent ileostomy.

\section{Other Surgical Options for Ulcerative Colitis}

Due to an often long-standing perirectal inflammatory reaction, surgical dissection may be hampered, and erectile dysfunction after IPAA occurs in 13-38.6\% [2, 17]. A meta-analysis from 2011 showed on top of an already preoperatively existing infertility rate of $20 \%$ an increase to up to $63 \%$ after IPAA [18]. In this analysis, the fertility was significantly reduced after IPAA for UC as well as for patients with FAP. Therefore, in young patients also without proctitis a subtotal colectomy with an ileal-rectal anastomosis can be considered. These patients must be made aware of the possible need for completing proctectomy in the further course.

In principle, a higher patient age is not a contraindication for an IPAA. In view of the increased stool frequency due to relative sphincter weakness and a significantly higher postoperative morbidity, however, a terminal ileostomy has to be discussed with these patients as the most appropriate concept.

The continent Kock ileostomy is a technically complex surgical procedure. It is constructed from the last $45 \mathrm{~cm}$ of the ileum by creating a reservoir below the abdominal wall with invagination of the reservoir outlet. The invagination of the ileal outlet generates a valve mechanism that enables the continence of the reservoir. The procedure is associated with a high complication rate and is only an option in exceptional situations [19].

\section{Perioperative Medication in Patients with \\ Inflammatory Bowel Disease}

Due to the heterogeneity of patients with inflammatory bowel disease (IBD), with mostly multimodal drug therapy and changing concepts, there is inconsistent data about perioperative medication that mainly consists of retrospective studies. Several studies do not differentiate between UC and Crohn's disease but examine the perioperative risk in IBD in general. However, sufficient data exist about the perioperative impact of corticosteroids. The German S3 guideline on UC suggests tapering the glucocorticoid dose preoperatively [3]. There is a clear relationship between perioperative medication and complication rate [20]. A preoperative reduction to $<20 \mathrm{mg}$ of prednisolone equivalent is desirable in any case. Most studies did not show an influence of thiopurines on postoperative septical complications; however, there is data about increased septical complications after thiopurine usage in Crohn's disease [21]. Based on this observation we discontinue thiopurine therapy 3 days preoperatively also in UC. Meta-analyses about the risk of perioperative anti-TNF-alpha therapy have shown increased infectious and non-infectious complication rates [22]. Therefore, a discontinuation of therapy should be aimed for at least 2 weeks prior to surgery. Finally, interdisciplinary management for the best timing of surgery plays an indispensable role.

\section{Conclusion}

Restorative proctocolectomy with IPAA represents the surgical treatment of choice for UC. Chronic pouchitis is one of the main factors that limit the surgical success of curing UC. Despite a considerable postoperative complication rate, normal quality of life can be achieved with a long-term pouch success rate of $>90 \%$ after 10 and 20 years of follow-up in experienced centers. A close cooperation between the various disciplines in the pre- and postoperative setting is essential for an optimal outcome of patients with UC.

\section{Disclosure Statement}

Prof. Klar is scientific adviser to Takeda Pharma. None of the authors has a conflict of interest regarding the contents of this article.

\section{References}

1 Molodecky NA, Soon IS, Rabi DM, et al: Increasing incidence and prevalence of the inflammatory bowel diseases with time, based on systematic review. Gastroenterology 2012;142:46-54.

2 Frolkis AD, Dykeman J, Negrón ME, et al: Risk of surgery for inflammatory bowel diseases has decreased over time: a systematic review and meta-analysis of population-based studies. Gastroenterology 2013;145: 996-1006.
Dignass A, Preiss JC, Aust DE, et al: Updated German guideline on diagnosis and treatment of ulcerative colitis, 2011 (Article in German). Z Gastroenterol 2011;49: 1276-1341.

4 Gan SI, Beck PL: A new look at toxic megacolon: an update and review of incidence, etiology, pathogenesis, and management. Am J Gastroenterol 2003;98:23632371.

5 Eaden JA, Abrams KR, Mayberry JF: The risk of colorectal cancer in ulcerative colitis: a meta-analysis. Gut 2001;48:526-535.
6 Devaraj B, Kaiser AM: Surgical management of ulcerative colitis in the era of biologicals. Inflamm Bowel Dis 2015;21:208-220.

7 Singh P, Bhangu A, Nicholls RJ, et al: A systematic review and meta-analysis of laparoscopic vs open restorative proctocolectomy. Colorectal Dis 2013; 15:e340351.

8 Fazio VW, Kiran RP, Remzi FH, et al: Ileal pouch anal anastomosis: analysis of outcome and quality of life in 3707 patients. Ann Surg 2013;257:679-685. 
9 Kaplan GG, McCarthy EP, Ayanian JZ, et al: Impact of hospital volume on postoperative morbidity and mortality following a colectomy for ulcerative colitis. Gastroenterology 2008;134:680-687.

10 Stocchi L, Pemberton JH: Pouch and pouchitis. Gastroenterol Clin North Am 2001;30:223-241.

11 Batista D, Raffals L: Role of intestinal bacteria in the pathogenesis of pouchitis. Inflamm Bowel Dis 2014;20: 1481-1486.

12 Landy J, Al-Hassi HO, McLaughlin SD, et al: Etiology of pouchitis. Inflamm Bowel Dis 2012;18:1146-1155.

13 Gionchetti P, Rizzello F, Venturi A, et al: Antibiotic combination therapy in patients with chronic, treatment-resistant pouchitis. Aliment Pharmacol Ther 1999;13:713-718.

14 Holubar SD, Cima RR, Sandborn WJ, et al: Treatment and prevention of pouchitis after ileal pouch-anal anastomosis for chronic ulcerative colitis. Cochrane Database Syst Rev 2010;6:CD001176.

15 Ikeuchi H, Uchino M, Matsuoka H, et al: Surgery for ulcerative colitis in 1,000 patients. Int J Colorectal Dis 2010;25:959-965.
6 Heuschen UA, Schmidt J, Allemeyer E, et al: The ileoanal pouch procedure: complications, quality of life, and long-term results (Article in German). Zentralbl Chir 2001;126:36-42.

17 Johnson P, Richard C, Ravid A, et al: Female infertility after ileal pouch-anal anastomosis for ulcerative colitis. Dis Colon Rectum 2004;47:1119-1126.

18 Rajaratnam SG, Eglinton TW, Hider P, et al: Impact of ileal pouch-anal anastomosis on female fertility: metaanalysis and systematic review. Int J Colorectal Dis 2011;26:1365-1374.

19 Aytac E, Ashburn J, Dietz DW: Is there still a role for continent ileostomy in the surgical treatment of inflammatory bowel disease? Inflamm Bowel Dis 2014; 12:2519-2525.

20 Subramanian V, Saxena S, Kang JY, et al: Preoperative steroid use and risk of postoperative complications in patients with inflammatory bowel disease undergoing abdominal surgery. Am J Gastroenterol 2008;103: 2373-2381.
21 Myrelid P, Olaison G, Sjödahl R, et al: Thiopurine therapy is associated with postoperative intra-abdominal septic complications in abdominal surgery for Crohn's disease. Dis Colon Rectum 2009;52:13871394.

22 Narula N, Charleston D, Marshall JK: Meta-analysis: peri-operative anti-TNFa treatment and post-operative complications in patients with inflammatory bowel disease. Aliment Pharmacol Ther 2013;37:10571064.

23 Michelassi F, Lee J, Rubin M, et al: Long-term functional results after ileal pouch anal restorative proctocolectomy for ulcerative colitis: a prospective observational study. Ann Surg 2003;238:433-441.

24 McGuire BB, Brannigan AE, O'Connell PE, et al: Ileal pouch-anal anastomosis. Br J Surg 2007;94:812-823. 